\title{
Pontos de adesão na cirurgia do contorno corporal
}

\author{
Adhesion suture in plastic of body countering
}

Ricardo Frota BogGio ${ }^{1}$

FÁbio Rosa de Almeida RICARDO BAROUDI ${ }^{2}$

Trabalho realizado no Serviço de Cirurgia Plástica Oswaldo Cruz, Instituto Brasileiro de Pesquisa e Ensino, São Paulo, SP, Brasil.

Trabalho apresentado para ascensão à categoria de membro titular da SBCP.

Artigo submetido pelo SGP (Sistema de Gestão de Publicações) da RBCP.

Artigo recebido: 7/1/2011

Artigo aceito: 21/3/2011

\section{RESUMO}

Introdução: Seromas, hematomas e alterações cicatriciais são complicações potenciais no pós-operatório das abdominoplastias. Drenagem aspirativa e pontos de adesão são estratégias utilizadas na prevenção destas complicações. A proposta deste estudo é avaliar a eficácia dos pontos de adesão na prevenção ou redução da formação de seroma, hematoma e de complicações cicatriciais em pacientes submetidos a cirurgia plástica do contorno corporal. Método: Cento e quinze pacientes (grupo 2: janeiro de 2006 - junho de 2010) submetidos a cirurgia plástica do contorno corporal com o uso dos pontos de adesão foram comparados, retrospectivamente, com um grupo de 39 pacientes (grupo 1: janeiro de 2004 - dezembro de 2005) submetidos a mesma cirurgia com o uso de drenagem aspirativa. Avaliação clínica dos pacientes foi realizada com 7 dias, 2 semanas, um, dois, três e seis meses de pós-operatório. Resultados: No grupo 1, houve 9 (23\%) casos de seroma, $1(2,5 \%)$ de hematoma e $20(55 \%)$ de complicações cicatriciais, enquanto que, no grupo 2 , nenhum caso de seroma ou hematoma foi reportado. A avaliação clínica revelou diferenças estatísticas entre o uso dos pontos de adesão e a drenagem aspirativa, no que se refere à incidência de seroma e de complicações cicatriciais. Conclusão: O uso dos pontos de adesão é um método eficiente na prevenção de seromas, hematomas e complicações cicatriciais nas cirurgias do contorno corporal.

Descritores: Abdome. Seroma/prevenção \& controle. Cirurgia plástica/métodos.

\begin{abstract}
Introduction: Seroma, hematoma and wound alterations are potential complications following abdominoplasty. Aspiratory drainage and adhesion suture are widely employed strategies for prevention of these complications. The purpose of this study is to evaluate the efficacy of adhesion suture in preventing or reducing seroma, hematoma formation and local wound complications in patients undergoing plastic of body countering. Methods: 115 patients (group 2: January 2006 - June 2010) who underwent plastic of body countering with the use of adhesion suture were retrospectively compared with a group of 39 patients (group 1: January 2004 - December 2005) who underwent with the use aspiratory drainage. Clinical evaluation of the patients was performed seven days, two weeks, one, two, three and six months postoperatively. Results: In group 1, there was 9 (23\%) cases of seroma, 1 $(2.5 \%)$ case of hematoma and $20(55 \%)$ cases of wound complications, whereas in group 2 no seroma or hematoma was reported. In this group, there were $14(12 \%)$ cases of wound complications. Clinical evaluation revealed statistical difference between the use of adhesion suture and the aspiratory drainage in the incidence of seroma and wound complications. Conclusion: The use of adhesion suture is an effective measure to prevent seroma, hematoma and wound complications in patients undergoing plastic of body countering.
\end{abstract}

Keywords: Abdomen. Seroma/prevention \& control. Plastic surgery/methods.

\footnotetext{
1. Membro Titular da Sociedade Brasileira de Cirurgia Plástica (SBCP), Doutor em Ciências pela Universidade de São Paulo (USP); Membro do corpo docente do Serviço de Cirurgia Plástica Oswaldo Cruz e do Instituto Brasileiro de Pesquisa e Ensino, São Paulo, SP, Brasil.

2. Membro Titular da SBCP; Proprietário da Clínica de Cirurgia Plástica Dr. Fábio Rosa, São Paulo, SP, Brasil.

3. Membro Titular da SBCP, Editor da Revista Brasileira de Cirurgia Plástica, São Paulo, SP, Brasil.
} 


\section{INTRODUÇÃO}

Os 130 anos de história das abdominoplastias, publicados por Avelar ${ }^{1}, \mathrm{Hakme}^{2}$ e Baroudi ${ }^{3}$, entre outros autores, registram inúmeros procedimentos técnicos, face às diversificações morfológicas que esta região do tronco sofre no decurso da vida. Atualmente, as abdominoplastias podem ser divididas em 4 grupos básicos: 1. abdominoplastia pubiana transversal baixa ${ }^{4}, 2$. abdominoplastia xifopúbica com compensação transversal pubiana, com cicatriz resultante em $\mathrm{T}$ invertido ${ }^{5}$, 3. miniabdominoplastias ampliada e não ampliada, com incisão tipo Pffanestiel alongada ${ }^{6,7} \mathrm{e}$ 4. abdominoplastia reversa ${ }^{8}$. Ainda dentro da evolução das abdominoplastias, Gonzalez-Ulloa ${ }^{9}$, em 1960, descreveu a abdominoplastia circular.

Ao longo dos anos, as abdominoplastias têm passado por contínuos refinamentos técnicos que têm contribuído na execução do procedimento, na redução do número de complicações e na obtenção de melhores resultados em longo prazo, sempre com o compromisso da reprodutibilidade, como tem sido constantemente observado na literatura especializada.

O índice de complicações, no pós-operatório das abdominoplastias, é extremamente variado, com valores oscilando entre 4 e $80 \%{ }^{10}$. Uma das mais frequentes complicações, encontradas nestes pós-operatórios, é a ocorrência de seromas, cuja incidência varia entre 10 e $15 \%$. Nas últimas décadas, classicamente, tem-se utilizado drenos de Penrose ${ }^{\circledR}$ ou a vácuo na prevenção dos seromas. Apesar do uso rotineiro dos drenos, vários são os trabalhos que continuam a descrever a ocorrência desta complicação no pós-operatório das cirurgias abdominais ${ }^{11-13}$.

Baroudi e Ferreira ${ }^{14,15}$, em 1996 e 1998, publicaram seus resultados sobre a aplicação dos pontos de adesão no decurso das abdominoplastias e em demais áreas dissecadas, eliminando o espaço morto e a possibilidade de ocorrência de hematomas e seromas.

O objetivo do presente trabalho é relatar a experiência dos autores com a utilização dos pontos de adesão na cirurgia do contorno corporal, especificamente nas abdominoplastias isoladas e circulares.

\section{MÉTODO}

No período de janeiro de 2004 a junho de 2010, foram realizadas 154 abdominoplastias associadas ou não a outros procedimentos cirúrgicos. A idade das pacientes variou entre 34 a 65 anos, com média de 45 anos, sendo 151 pacientes do sexo feminino e 3 do masculino. Todos os pacientes foram submetidos a anamnese e exame físico detalhado, exames laboratoriais e registro fotográfico. Pacientes portadores de comorbidades não compensadas, índice de massa corporal superior a 35 e tabagismo importante tiveram suas cirurgias contraindicadas. Todos foram esclarecidos sobre os procedimentos, intercorrências, complicações e resultados esperados. Todos receberam informativos impressos sobre o procedimento proposto e assinaram termos de consentimento informado e de responsabilidade.

A seleção do tipo de cirurgia foi determinada pelo grau de comprometimento do contorno corporal ${ }^{16}$. Pacientes com comprometimento da parede anterior do abdome, predominantemente infraumbilical, foram submetidos à miniabdominoplastias, ampliadas ou não; pacientes com comprometimento dermoadiposo infra e supraumbilical foram submetidos à abdominoplastia por incisão pubiana transversal baixa, enquanto que os pacientes que apresentavam importante excedente de tecido dermoadiposo, normalmente secundário a emagrecimento intenso, foram operados pela técnica xifopúbica, também chamada "flor-de-lis" ou em T invertido. Finalmente, os pacientes com excessos de panículo dermoadiposo, com extensão para os flancos e regiões glúteas, foram submetidos à corpoplastia circular.

As cirurgias realizadas durante o período de 2004 a 2005 (grupo 1) foram drenadas com Portovac $\AA \mathrm{n}^{\circ} 4.8$, tendo o mesmo permanecido até o volume drenado ter sido inferior a $30 \mathrm{ml}$, em 24 horas. Em todos os pacientes operados de abdominoplastias e corpoplastias durante o período de 2006 a 2010 (grupo 2), foram aplicados pontos de adesão sem a inclusão de drenos aspirativos.

\section{Técnica Cirúrgica}

As cirurgias foram realizadas sob anestesia geral ou epidural com sedação.

Em todos os pacientes, foi utilizada compressão dos membros inferiores por meias elásticas e massageador pneumático intermitente, sendo o uso seletivo de heparina de baixo peso molecular $(40 \mathrm{mg} / 7$ dias $)$ indicado de acordo com classificação individual de risco.

No decurso das miniabdominoplastias e abdominoplastias, após os tempos de dissecção e ressecção dos excessos cutâneos, foi realizada a plicatura dos músculos retos, quando indicada, e, posteriormente, o reposicionamento do retalho dermoadiposo por intermédio da aplicação pontos isolados de adesão, sendo, para tanto, utilizado fio absorvível 2-0, em linhas e colunas a partir do limite superior da dissecção junto ao xifoide, a distância máxima de $4 \mathrm{~cm}$ entre eles, até a sutura das bordas da incisão. Os pontos foram aplicados transfixando a fáscia muscular da parede abdominal e a camada adiposa lamelar, com ou sem inclusão da fáscia superficial. No nível de exteriorização, o umbigo foi posicionado no plano cutâneo.

A cada linha de sutura, manobras para avaliação do adequado posicionamento do retalho foram realizadas, até a junção com a borda inferior da incisão cutânea. $\mathrm{O}$ número de pontos variou de 40 a 45, em média. As bordas da incisão 
foram suturadas, nos planos adiposo e dérmico, com pontos isolados de fio absorvível 3-0, sendo a camada cuticular suturada com fio não-absorvível 4-0, aplicado de maneira contínua (Figuras 1 e 2).

Nas abdominoplastias realizadas pela técnica xifopúbica, após a ressecção do retalho dermoadiposo, constituído por segmento horizontal e vertical (Figuras 3A e 3B), foi realizada a plicatura dos músculos reto abdominais com fio não-absorvível 0 , com pontos isolados e, posteriormente, uma sutura de reforço com fio não-absorvível 2-0, aplicada de maneira contínua. Na sequência, foi realizado o reposicionamento dos retalhos dermoadiposos por meio da aplicação dos pontos de adesão, fixando a camada lamelar à aponeurose muscular, de tal forma a tracionar as bordas do retalho em direção à linha média (Figuras $3 \mathrm{C}$ a $3 \mathrm{~F}$ ).

Nas corpoplastias, foi dada preferência à anestesia geral, com entubação endotraqueal, sendo a cirurgia iniciada com o paciente posicionado em decúbito ventral horizontal. Após as devidas ressecções (Figuras 4A a 4C), foi realizada a síntese da área cruenta com pontos isolados de adesão, a cada $4 \mathrm{~cm}$, com fio absorvível 2-0, entre a aponeurose dos músculos glúteos e a camada lamelar do retalho dermoadiposo (Figuras 4D e 4E). As bordas da incisão foram suturadas com pontos isolados de fio absorvível 3-0, aplicados de forma a incluir a fáscia superficial. A sutura dérmica também foi realizada com pontos isolados de fio absorvível 3-0, sendo a sutura cuticular realizada com fio não-absorvível 4.0, aplicado de maneira contínua (Figura 4F). Após o término desta primeira

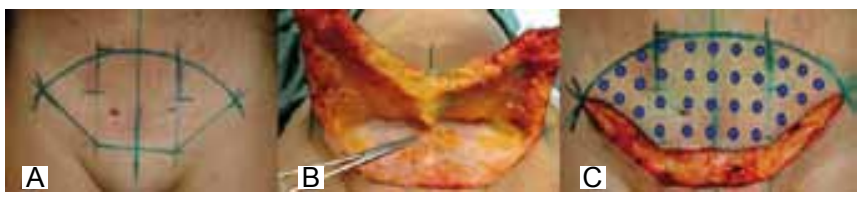

Figura 1 - Transoperatório de miniabdominoplastia. A: Marcação; B: Pontos de adesão sendo aplicados;

C: Projeção dos pontos de adesão no retalho dermoadiposo.

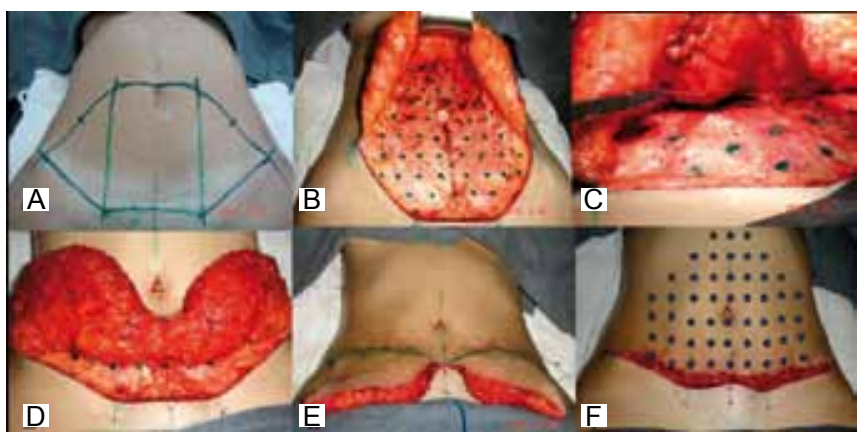

Figura 2 - Transoperatório de abdominoplastia pubiana transversal baixa evidenciando os pontos de adesão.

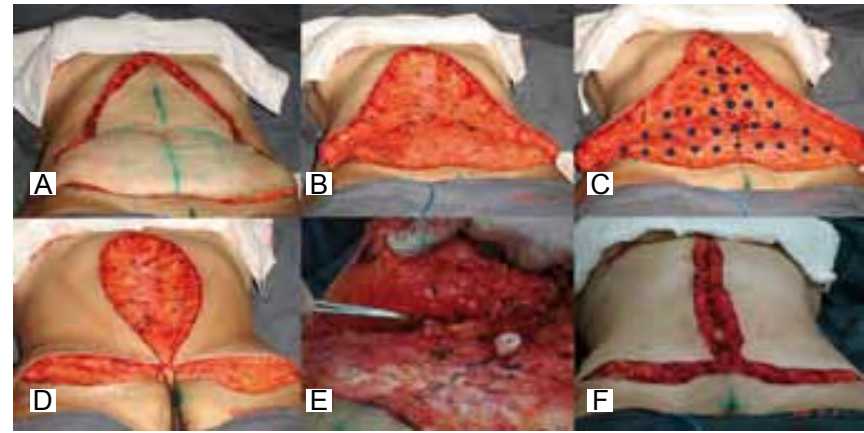

Figura 3 - Transoperatório de abdominoplastia em "Flor-de-Lis". $\boldsymbol{A}$ e B: Dissecção do retalho dermoadiposo; $\boldsymbol{C}$ : Programação dos pontos de adesão; $\boldsymbol{D}$ e $\boldsymbol{E}$ : Reposicionamento das bordas cirúrgicas com tração dos retalhos em direção à linha média do abdome;

$\boldsymbol{F}$ : Retalhos reposicionados e fixos pelos pontos de adesão.

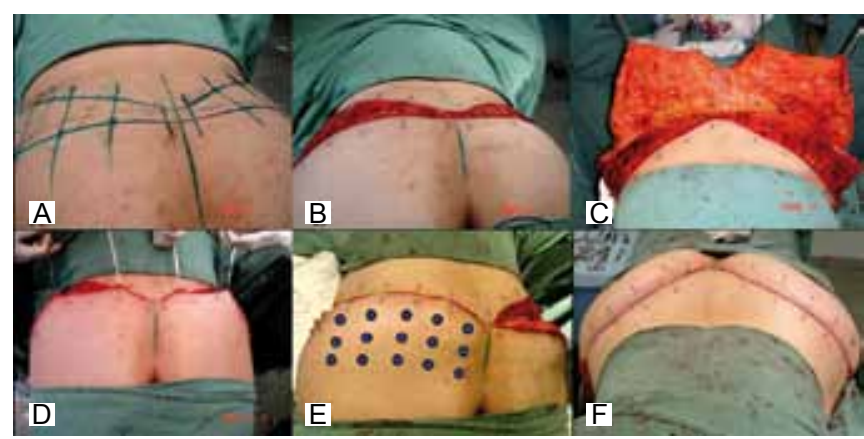

Figura 4 - Transoperatório de abdominoplastia circular, com o paciente posicionado em decúbito ventral horizontal.

$\boldsymbol{A}-\boldsymbol{C}$ : Dissecção do retalho dermoadiposo ( $\boldsymbol{C}$ - vista cranial);

$\boldsymbol{D}$ e $\boldsymbol{E}$ : Reposicionamento do retalho dermoadiposo e fixação com pontos de adesão, $\boldsymbol{F}$ : Aspecto final (vista cranial).

fase da cirurgia, os excessos cutâneos dos flancos foram isolados e cobertos, e o paciente posicionado em decúbito dorsal horizontal. O tempo da abdominoplastia foi então realizado dentro da rotina descrita, sendo, posteriormente, ressecados os excessos laterais e suturado bilateralmente, resultando em uma cicatriz circular. Ao término da cirurgia, as cicatrizes foram imobilizadas com esparadrapo poroso $\mathrm{e}$ o paciente vestido com cinta modeladora.

\section{Cuidados Pós-operatórios}

A antibioticoterapia, iniciada no transoperatório (cefalosporina $1 \mathrm{~g}$, a cada 3 horas), foi mantida no pós-operatório por uma semana, na dosagem de $500 \mathrm{mg}$ duas vezes ao dia. Quando indicada, a heparina de baixo peso ( $40 \mathrm{mg} / \mathrm{dia})$ foi utilizada até o $7^{\circ}$ dia de pós-operatório. 
O massageador pneumático intermitente, utilizado durante o procedimento cirúrgico, foi mantido por 24 horas, sendo o uso das meias elásticas indicado até o retorno da deambulação ativa.

No pós-operatório, as pacientes foram reavaliadas após 7 dias, duas semanas, um, dois, três e seis meses. Foram registrados dados sobre presença de hematomas, seromas, cicatrizes inestéticas e mal posicionadas, além do índice de satisfação da paciente e da equipe cirúrgica.

\section{Análise Estatística}

O teste de $\mathrm{t}$ de Student foi utilizado para avaliação da diferença entre dois grupos. O software utilizado para a análise foi o Graphpad Prism (GraphPad Software, Inc., San Diego, CA, USA).

\section{RESULTADOS}

Durante o período 2004-2005, foram operados 39 pacientes, sendo apenas um do sexo masculino. Um $(2,5 \%)$ paciente foi submetido à miniabdominoplastia, $10(25 \%)$ à abdominoplastia tipo transversal baixa e $2(5 \%)$ à abdominoplastia em "flor-de-lis"; 16 (41\%) cirurgias foram associadas à lipoaspiração dos flancos e dorso e 10 (25\%), à mamoplastia.

Em nenhum dos casos operados, foi registrada intercorrência anestésica; no $14^{\circ}$ dia de pós-operatório, 1 $(2,5 \%)$ dos pacientes apresentou quadro de trombose venosa profunda (TVP), permanecendo internado por 10 dias, com boa evolução.

Todos os pacientes operados neste período tiveram suas cirurgias drenadas por Portovac ${ }^{\circledR} 4.8$, o qual foi mantido até o volume drenado ser igual a $30 \mathrm{ml}$, em 24 horas. O tempo mínimo de permanência do dreno foi de 2 e máximo de 8 dias, tendo como média 5 dias.

Dos 39 pacientes, 9 (23\%) apresentaram seroma após a remoção do dreno, sendo 7 (77\%) aspirados por punção transcutânea e $2(22 \%)$ reoperados para a remoção da bolsa serosa. Dentre os pacientes com seroma, 4 (44\%) haviam sido submetidos a abdominoplastia isolada, 3 (33\%) associada à lipoaspiração dos flancos e dorso e $2(22 \%)$ à mamoplastia. Um único $(2,5 \%)$ paciente apresentou hematoma de pequeno volume no hipogástrio, com resolução espontânea, sem a necessidade de intervenção. Em 12 (30\%) pacientes, houve deiscência da cicatriz pubiana, sendo que, em 10 (83\%), a cicatrização evoluiu por segunda intenção e em 2 (16\%), optou-se pela ressutura, dada a extensão da deiscência. Seis $(15 \%)$ pacientes apresentaram cicatrizes hipertróficas e $2(5 \%)$, cicatrizes queloideanas. Cicatrizes mal posicionadas foram observadas em $16(41 \%)$ dos pacientes operados no período, gerando insatisfação aos mesmos e à equipe cirúrgica. Destes, $10(62 \%)$ tiveram as cicatrizes retocadas e $6(38 \%)$ preferiram não ser submetidos a novo procedimento para a correção da cicatriz. Em nenhum dos pacientes operados ocorreu necrose cutânea ou do pedículo do umbigo (Tabela 1 e Figura 5).

Tabela 1 - Cirurgias e principais intercorrências de pósoperatório.

\begin{tabular}{l|c|c}
\hline \multicolumn{1}{c|}{ Característica } & 2004-2005 & 2006-2010 \\
\hline Cirurgias realizadas (total) & 39 & 115 \\
\hline Miniabdominoplastia & 1 & 5 \\
\hline Abdominoplastia & 10 & 32 \\
\hline Abdominoplastia/lipoaspiração & 16 & 49 \\
\hline Abdominoplastia/mamoplastia & 10 & 19 \\
\hline Abdominoplastia em "flor-de-lis" & 2 & 7 \\
\hline Abdominoplastia circular & 0 & 3 \\
\hline Drenagem (Portovac ${ }^{4}$ 4.8) & 39 & 0 \\
\hline Seromas & 9 & 0 \\
\hline Hematoma & 1 & 0 \\
\hline Necroses cutâneas & 0 & 0 \\
\hline Deiscências & 12 & 3 \\
\hline Cicatrizes hipertróficas & 6 & 6 \\
\hline Queloides & 2 & 5 \\
\hline Cicatrizes mal posicionadas & 16 & 23 \\
\hline TVP / TEP & 1 & 2 \\
\hline
\end{tabular}

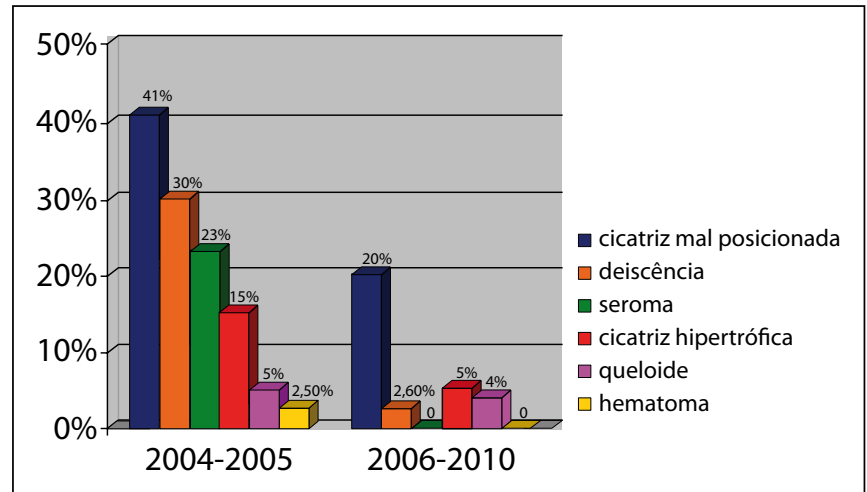

Figura 5 - Comparação da incidência (\%) das principais complicações entre os periodos 2004-2005 (sem pontos de adesão) e 2006-2010 (com pontos de adesão) $(p<0,0001)$. 
No período de janeiro de 2006 a junho de 2010, 115 pacientes foram operados, sendo $113(98 \%)$ do sexo feminino. Cinco (4\%) pacientes foram submetidos a miniabdominoplastia, 32 (27\%), somente a abdominoplastia, 49 (42\%), a abdominoplastia associada à lipoaspiração de flancos e dorso e $19(16 \%)$, a abdominoplastia combinada à mamoplastia. Sete $(6 \%)$ pacientes foram submetidos à abdominoplastia em "flor-de-lis" e 3 (2,6\%), a corpoplastia, também chamada abdominoplastia circular. Dos 115 pacientes, 113 (98\%) evoluíram no trans e no pós-operatório sem intercorrências clínicas, sendo observado tromboembolismo pulmonar(TEP) em $2(1,7 \%)$ deles. Um dos pacientes com TEP havia sido submetido à abdominoplastia e o outro à abdominoplastia associada à lipoaspiração. Ambos pacientes necessitaram de cuidados intensivos, com evolução sem consequências.

Os pacientes operados no referido período não tiveram suas cirurgias drenadas por sucção a vácuo ou por qualquer outro sistema de drenagem, sendo utilizado nestes pacientes, exclusivamente, pontos de adesão. Em nenhum dos casos operados foi observada, clinicamente, a presença de seroma ou hematoma que justificasse drenagem percutânea ou aberta. Nenhum dos pacientes operados apresentou necrose de retalho cutâneo ou do pedículo do umbigo, a exemplo dos pacientes operados no período 2004-2005. Três (2,6\%) pacientes evoluíram com deiscência da sutura, sendo uma em flanco, após a realização de abdominoplastia circular, necessitando de ressutura, e duas em compensação vertical em forma de "T invertido", na linha média do púbis, cicatrizando por segunda intenção. Após 3 meses, estes dois pacientes foram submetidos a retoque local da cicatriz. Cicatrizes hipertróficas foram diagnosticas em $6(5 \%)$ pacientes e queloideanas em $5(4 \%)$. O posicionamento da cicatriz no púbis foi considerado satisfatório em $80 \%$ dos pacientes operados com ponto de adesão. Dentre os 23 pacientes com cicatriz mal posicionada, 7 (39\%) tiveram suas cicatrizes retocadas cirurgicamente (Tabela 1 e Figura 5).

\section{DISCUSSÃO}

A ocorrência de hematomas e seromas é a complicação mais frequente observada no pós-operatório das abdominoplastias. $\mathrm{O}$ mecanismo proposto na sua gênese baseia-se nas lesões vasculares e linfáticas, no desenvolvimento de espaço morto secundário ao descolamento do retalho dermoadiposo e na força de cisalhamento existente entre o retalho e o complexo músculo-aponeurótico ${ }^{17}$. Bozola e Psillakis ${ }^{16}$, em 1988, relacionaram a incidência de seromas com a extensão da cirurgia, tendo as cirurgias de maior porte predisposição aumentada ao desenvolvimento desta complicação. Matarasso $^{18}$, em 1991, correlacionou a incidência de seromas ao tipo de cirurgia empregada, sugerindo descolamentos limitados e a utilização de drenos de sucção. Em 1999,
Vastine et al. ${ }^{19}$ analisaram, retrospectivamente, 90 pacientes submetidos a abdominoplastia, observando a ocorrência de complicações em $80 \%$ dos pacientes que se apresentavam obesos na época da cirurgia, sendo as complicações mais frequentes seromas e deiscências cicatriciais. Nos pacientes com peso normal, a ocorrência de complicações foi de $30 \%$. Nurkim et al. ${ }^{20}$, em 2002, relataram a ocorrência de seromas em $57 \%$ dos pacientes submetidos a abdominoplastias sem inclusão de drenos, em $29 \%$ dos pacientes submetidos à mesma cirurgia associada a drenagem tubular e em $4 \%$ dos pacientes submetidos a drenagem a vácuo. Baroudi e Ferreira $^{14}$, em 1998, descreveram a utilização de pontos de adesão, com fios absorvíveis, entre o retalho dermoadiposo e a aponeurose muscular para diminuir o "espaço morto" após o descolamento nas abdominoplastias. Daltrey et al. ${ }^{21}$, em 2006, em estudo randomizado envolvendo 108 pacientes submetidas à reconstrução mamária com grande dorsal, observaram redução significativa na incidência e no volume de seromas no pós-operatório. Moura \& Bezerra ${ }^{22}$, em 2008, observaram resultados semelhantes ao avaliar, retrospectivamente, 20 pacientes submetidas à reconstrução mamária com TRAM. Nahas et al. ${ }^{23}$, em 2007, avaliaram com ultrassonografia 21 pacientes submetidos à abdominoplastia e pontos de adesão, observando a presença de seromas, com volume médio de $8,2 \mathrm{ml}$, duas semanas após a realização da cirurgia.

No presente trabalho, foi possível observar que, nos anos de 2004 e 2005, 23\% dos pacientes submetidos a cirurgias do contorno corporal apresentaram seromas após a remoção do dreno aspirativo, enquanto que, entre os pacientes operados nos anos de 2006 a 2010, nos quais foram aplicados os pontos de adesão, não houve ocorrência de nenhum caso de seroma, confirmando os conceitos de Baroudi \& Ferreira ${ }^{14,15}$. Com o advento dos pontos de adesão foi possível reduzir o "espaço morto" gerado pelo descolamento do retalho dermoadiposo, além de diminuir a capacidade de deslizamento do retalho sobre o plano aponeurótico, excluindo, assim, dois dos principais fatores predisponentes envolvidos no desenvolvimento de seromas e hematomas no pós-operatório. A realização de rigorosa hemostasia, aliada ao cuidado de se evitar a manipulação excessiva do retalho e a realização de cirurgias combinadas, são condutas importantes que devem ser associadas aos pontos de adesão na profilaxia dos seromas.

A fixação do retalho dermoadiposo ao plano aponeurótico, pelos pontos de adesão, proporciona ainda importante redução das forças de tração, exercida pelo retalho sobre a cicatriz pubiana, diminuindo, de maneira significativa, a prevalência de deiscências e de cicatrizes mal posicionadas e alargadas, em comparação às cirurgias classicamente realizadas. A tração consiste também em um importante fator predisponente no desenvolvimento de cicatrizes hipertróficas, com a redução da tração exercida pelo retalho sobre a cicatriz pubiana foi possível observar incidência 
significativamente menor de cicatrizes hipertróficas, nos casos em que os pontos de adesão foram utilizados. A incidência de queloides, nas cirurgias com ou sem a utilização de pontos de adesão, não apresentou diferença significativamente estatística.

O tempo de hospitalização dos pacientes submetidos à cirurgia do contorno corporal, anteriormente determinado pelo volume coletado pelos drenos aspirativos, com a utilização dos pontos de adesão foi abreviado, em média, para 24 horas, possibilitando ao paciente um retorno mais precoce às atividades habituais.

A aplicação dos pontos de adesão tem aumentado, em média, em 30 minutos o tempo cirúrgico de nossas abdominoplastias. Partindo da premissa que, os benefícios proporcionados pelos pontos de adesão superam em muito as inconveniências geradas pelos seromas, hematomas e cicatrizes inestéticas, este aumento no tempo cirúrgico fica justificado.

A observação de que muitos cirurgiões plásticos ainda não utilizam os pontos de adesão ou, quando o utilizam acabam por aplicar um número reduzido de pontos, muitas vezes associando-os à inclusão de drenos, a realização do presente trabalho reforça as orientações preconizadas por Baroudi e Ferreira, colaborando na sedimentação e divulgação da técnica.

\section{CONCLUSÃO}

A utilização dos pontos de adesão na cirurgia do contorno corporal tem colaborado sensivelmente na redução de complicações, como seromas, hematomas, deiscências, cicatrizes mal posicionadas, alargadas e hipertróficas, além de proporcionar ao paciente condições para retornar às suas atividades habituais mais precocemente.

\section{REFERÊNCIAS}

1. Avelar JM. Abdominoplasty. Systematization of a technique without external umbilical scar. Aesthetic Plast Surg. 1978;2:141-51.

2. Hakme F. Lipectomia peri e supra-umbilical nas abdominoplastias. Rev Bras Cir. 1979;69:27.

3. Baroudi R. Abdominoplasty: historical review and personal references. Newsletter. Lipoplasty Society North America. 1993;10(3):8.
4. Monard P. La resection esthetique du ventre em tablier avec transplantation de lómbelique. In: Pauchet $\mathrm{V}$, ed. La practique chirurgicale illusttree. vol V. Paris:Gastón Doin;1930.

5. Dufourmentel C, Mouly R. Chirurgie Plastique. Collection medicochirurgicale. Paris:Flamarion;1959.

6. Glicenstein J. Difficulties of the surgical treatment of abdominal dermodystrophies. Ann Surg Plast. 1975;20(2):147-55.

7. Wilkinson TS, Swarzt BE. Individual modifications in the body contour surgery: the "limited" abdominoplasty. Plast Reconstr Surg. 1986;77(5):779-84

8. Rebello C, Franco T. Abdominoplasty through a submammary incision. Int Surg. 1977;62(9):462-3.

9. Gonzalez-Ulloa M. Belt lipectomy. Br J Plast Surg. 1960;13:179-86.

10. Floros C, Davis PK. Complications and long-term results following abdominoplasty: a retrospective study. Br J Plast Surg. 1991;44(3):190-4.

11. Antonetti JW, Antonetti AR. Reducing seroma in outpatient abdominoplasty: analysis of 516 consecutive cases. Aesthetic Surg J. 2010;30(3):418-25

12. Beer GM, Wallner H. Prevention of seroma after abdominoplasty. Aesthet Surg J. 2010;30(3):414-7.

13. Fang RC, Lin SJ, Mustoe TA. Abdominoplasty flap elevation in a more superficial plane: decreasing the need for drains. Plast Reconstr Surg. 2010;125(2):677-82.

14. Baroudi R, Ferreira CA. Seroma: how to avoid it and how to treat it. Aesthet Surg J. 1998;18(6):439-41.

15. Baroudi R, Ferreira CA. Contouring the hip and the abdomen. Clin Plast Surg. 1996;23(4):551-72.

16. Bozola AR, Psillakis JM. Abdominoplasty: a new concept and classification for treatment. Plast Reconstr Surg. 1988;82(6):983-93.

17. Andrades P, Prado A, Danilla S, Guerra C, Benitez S, Sepulveda S, et al. Progressive tension sutures in the prevention of postabdominoplasty seroma: a prospective, randomized, double-blind clinical trial. Plast Reconstr Surg. 2007;120(4):935-46.

18. Matarasso A. Abdominolipoplasty: a system of classification and treatment for combined abdominoplasty and suction-assisted lipectomy. Aesthetic Plast Surg. 1991;15(2):111-21.

19. Vastine VL, Morgan RF, Williams GS, Gampper TJ, Drake DB, Knox LK, et al. Wound complications of abdominoplasty in obese patients. Ann Plast Surg. 1999;42(1):34-9.

20. Nurkim MV, Mendonça LB, Martins PAM, Silva JLB, Martins PDE. Incidência de hematoma e seroma em abdominoplastia com e sem uso de drenos. Rev Soc Bras Plast. 2002;17(1):69-74.

21. Daltrey I, Thomson H, Hussien M, Krishna K, Rayter Z, Winters ZE. Randomized clinical trial of the effect of quilting latissimus dorsi flap donor site on seroma formation. Br J Surg. 2006;93(7):825-30.

22. Moura RMG, Bezerra FJF. Emprego dos pontos de adesão nas reconstruções mamárias com TRAM mais tela sintética. Rev Bras Cir Plast. 2008;23(3):153-7.

23. Nahas FX, Ferreira LM, Ghelfond C. Does quilting suture prevent seroma in abdominoplasty? Plast Reconstr Surg. 2007;119(3):1060-4. 